\title{
cis-Acting Sequences Required for Expression of the Divergently Transcribed Drosophila melanogaster Sgs-7 and $S g s-8$ Glue Protein Genes
}

\author{
ANNEMARIE HOFMANN, $\dagger$ MARK D. GARFINKEL, $\ddagger$ AND ELLIOT M. MEYEROWITZ* \\ Division of Biology 156-29, California Institute of Technology, Pasadena, California 91125
}

Received 26 November 1990/Accepted 7 March 1991

\begin{abstract}
The $S g s-7$ and $S g s-8$ glue genes at $68 \mathrm{C}$ are divergently transcribed and are separated by 475 bp. Fusion genes with $A d h$ or lac $Z$ coding sequences were constructed, and the expression of these genes, with different amounts of upstream sequences present, was tested by a transient expression procedure and by germ line transformation. A cis-acting element for both genes is located asymmetrically in the intergenic region between -211 and $\mathbf{- 4 3}$ bp relative to $\mathbf{S g s}-\mathbf{7}$. It is required for correct expression of both genes. This element can confer the stageand tissue-specific expression pattern of glue genes on a heterologous promoter. An 86-bp portion of the element, from -133 to -48 bp relative to $S g s-7$, is shown to be capable of enhancing the expression of a truncated and therefore weakly expressed $\mathrm{Sgs}-3$ fusion gene. Recently described common sequence motifs of glue gene regulatory elements (T. Todo, M. Roark, K. Vijay Raghavan, C. A. Mayeda, and E. M. Meyerowitz, Mol. Cell. Biol. 10:5991-6002, 1990) are located within this 86-bp region.
\end{abstract}

The salivary gland secretion $(S g s)$, or glue protein, genes expressed in the salivary glands of Drosophila melanogaster third-instar larvae represent an example of a coordinately expressed set of genes. The set codes for proteins that are synthesized in one tissue and at one time in development $(1$, $25,26)$. The proteins are secreted into the lumen of the salivary gland and expelled just prior to pupariation to affix the pupa to a surface during metamorphosis $(12,27)$. Seven genes are known to code for components of the glue. The $S g s-3, S g s-7$, and $S g s-8$ genes are clustered within a 5,000-bp segment on the left arm of chromosome 3 at $68 \mathrm{C}$, while the $S g s-1, S g s-4, S g s-5$, and $S g s-6$ genes each reside at a different location in the genome. All of the chromosomal sites with glue genes correspond to regions showing large intermolt polytene chromosome puffs during the time of glue protein synthesis.

The $68 \mathrm{C}$ intermolt puff is interesting because of the clustering of the three genes found there. The order of the genes is telomere, $S g s-8, S g s-7, S g s-3$, centromere with respect to the left arm of the third chromosome. Transcription of $S g s-8$ proceeds leftward, transcription of $S g s-7$ proceeds rightward, and transcription of $S g s-3$ proceeds rightward $(16,40)$. The $5^{\prime}$ end of $S g s-8$ is separated from the $5^{\prime}$ end of $S g s-7$ by 475 bp of nontranscribed intergenic sequences (16), and the two genes are surrounded by two 285-bp elements that form an inverted repeat. The $3^{\prime}$ end of $S g s-7$ is separated from the $5^{\prime}$ end of $S g s-3$ by 1,958 bp (16, 40).

Two trans-acting regulators of this gene set have been described. The steroid hormone ecdysterone is required for both activation and cessation of RNA synthesis $(11,18)$, and the function eliminated by the lethal(1)nonpupariating-1 [l(1)npr-1] mutation is required for expression of the three $68 \mathrm{C}$ genes (10).

\footnotetext{
* Corresponding author.

$\dagger$ Present address: Institut für Genetik der Freien Universität Berlin, 1000 Berlin 33, Germany.

$\ddagger$ Present address: Department of Molecular Genetics and Cell Biology, The University of Chicago, Chicago, IL 60637.
}

Previous studies on the sequences required in cis for regulation of these genes have concentrated on those regulating the largest of the three genes, $S g s-3$, and have shown that there are at least three regulatory elements within the sequences upstream of the $S g s-3$ gene: the proximal element between -106 and $-56(34,46,57)$, the distal element between -629 and $-491(5,41,45,46)$, and multiple remote regions from 1.5 to $2.5 \mathrm{~kb}$ upstream (17). The proximal and distal elements are functionally equivalent and act synergistically to give high-level expression (46).

The experiments described here comprise an analysis of the $S g s-7 / S g s-8$ gene pair. As the genes are divergently transcribed, the general question is to determine the arrangement of cis-acting sequences required for each gene to function. Sequence analysis of the intergenic region has revealed conserved regions within the first $100 \mathrm{bp}$ upstream of $S g s-7$ and the first $100 \mathrm{bp}$ upstream of $S g s-8$ (16). These elements, the right copy and the left copy, respectively, contain sequences that resemble the ecdysterone regulatory element identified by Mestril et al. (39) in their study of the hsp23 gene.

We have identified the cis-acting regulatory sequences for $S g s-7$ and $S g s-8$ by reintroducing in vitro-manipulated genes either into somatic cells via the transient assay or somatic transformation procedure (35) or into the germ line of $D$. melanogaster via P-element-mediated transformation (47, 54). We have used gene fusion constructs of regulatory regions of $S g s-7$ and $S g s-8$ with the D. melanogaster Adh (alcohol dehydrogenase) gene or the Escherichia coli lacZ ( $\beta$-galactosidase) gene as reporter genes. These reporter gene choices were motivated by several considerations: first, to exploit sensitive histochemical reactions for the in situ detection of the protein products encoded by introduced fusion gene constructions; second, to exploit sensitive quantitative enzyme activity assays for measurement of gene function; third, to distinguish the gene products of newly introduced glue protein genes from the endogenous gene products, as there are no stocks that produce reduced amounts, or size variants, of $S g s-7$ or $S g s-8$ RNA or protein; and fourth, to enable us to test plasmid constructions simul- 
taneously for $S g s-7$ and $S g s-8$ gene function. The experiments confirm that a single 86-bp region functioning bidirectionally, located closer to the $S g s-7$ gene, is required for stage- and tissue-specific full expression of both genes. It contains sequences that have recently been identified as regulatory element consensus sequences for glue genes (55).

\section{MATERIALS AND METHODS}

Plasmid constructions. All plasmids were named according to the following convention: $\mathrm{G}=$ glue gene, $\mathrm{A}=A d h$ gene, $\mathrm{L}=$ lac $Z$ gene, $\mathrm{X}=$ xanthine dehydrogenase gene, $\mathrm{O}=$ synthetic oligonucleotide, $\mathrm{S}=S g s-4$ gene, and $\mathrm{C}=$ cisacting sequence.

Construction of the Sgs-7-Adh fusion gene. Plasmid pHAP (4) was used as the source of the promoterless D. melanogaster $\mathrm{Adh}$ gene for fusion to $\mathrm{Sgs-7}$ promoter sequences. The $A d h$ gene in this plasmid is modified by the addition of a HindIII linker in the $5^{\prime}$ untranslated region. A $1.8-\mathrm{kb}$ HindIII-XbaI fragment encompassing the $A d h$ gene was subcloned into pUC18. An 824-bp XbaI fragment containing the $S g s-7 / S g s-8$ intergenic region and portions of the $S g s-7$ and $S g s-8$ transcription units was cloned into the HindIII site after partially filling the HindIII and $X b a I$ termini. The plasmid was named pGAO-1, and the sequence of the joint between $S g s-7$ and $A d h$ was determined $(36,37)$. The junction between the two genes is in their $5^{\prime}$ untranslated regions. The $S g s-7$ contribution ends at +25 bp and the $A d h$ contribution begins at +13 bp relative to their respective transcripts. The $A d h$ contribution starts at +13 of the transcript that is initiated from the proximal promoter (3). Five nucleotides from the synthetic HindIII linker are sandwiched between the two Drosophila gene segments.

Construction of pGLAX1.0. The 2.2-kb fragment from pGAO-1 that contains the $S g s-7-A d h$ fusion gene was fused to custom-made synthetic 14-mer linker/adaptors and cloned into the EcoRI site of pOX4. Plasmid pOX4 (39a) contains $S g s-8$ sequences beginning in the $5^{\prime}$-flanking sequence and ending at the $B g$ lII site at +245 bp joined to the $E$. coli lacZ gene. The first 51 codons of the $S g s-8$ protein-coding region are joined to a synthetic-linker-derived codon, followed by the $E$. coli $\beta$-galactosidase-coding region beginning at the ninth codon. At the $3^{\prime}$ end of the lac $Z$ gene, a 268-bp segment of the $S g s-8$ gene contributes a polyadenylation site. The transformation vector pGLAX1.0 (Fig. 1) was constructed by cloning the $9.2-\mathrm{kb}$ SalI fragment that contained the $S g s-7-A d h$ and $S g s-8-l a c Z$ fusion genes into Carnegie 20 (48).

Construction of the pGLX and the pGAX series. The 4.2-kb $X b a I-K p n I$ fragment from pGLAX1.0 containing the entire Sgs-8-lacZ fusion gene was subcloned into pUC18 to give the starting clone for the pGLX series. The 2.2-kb EcoRI fragment from pGAZ-1 containing the $S g s-7-A d h$ gene was recloned into the vector fragment of pGAO-1 and served as the starting clone for the pGAX series. To remove DNA upstream of the fusion genes, BAL 31 digestions were used by setting up replica reactions that varied either the time of reaction or the concentration of BAL 31. The amount of DNA removed by BAL 31 was determined by digesting the reaction mixtures with the appropriate restriction enzymes and subjecting them to electrophoresis through a $2.5 \%$ agarose gel. The endpoints of the BAL 31 deletion molecules used in this study were determined by standard procedures for dideoxy sequencing $(49,56)$. Germ line transformation plasmids (Fig. 1) were constructed by recloning the promoter deletion molecules into the SalI site of Carnegie 20.
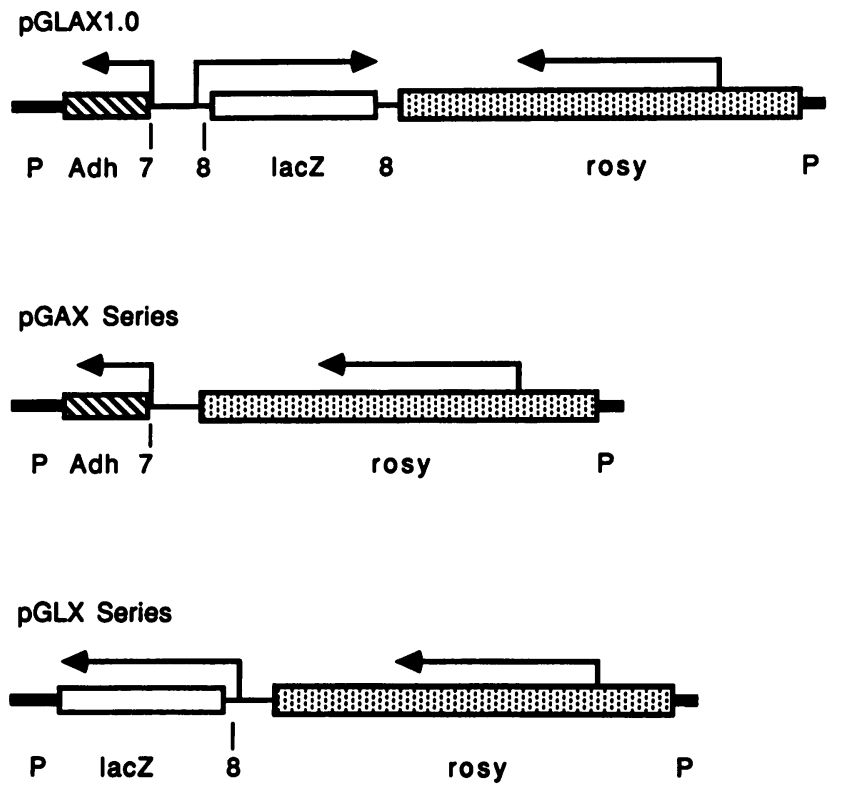

FIG. 1. Schematic representation of plasmids pGLAX1.0, pGAX, and pGLX. The origins of the various fragments are shown, as are the transcription directions of the different genes. P, Drosophila P element; Adh, Drosophila alcohol dehydrogenase gene; lacZ, E. coli $\beta$-galactosidase gene; rosy, Drosophila xanthine dehydrogenase gene; 7 , transcribed sequences from $\mathrm{Sgs}-7 ; 8$, transcribed and 3 ' sequences from $S g s-8$. The thin line between 7 and 8 represents the intergenic region of $\mathrm{Sgs}_{-7}$ and $\mathrm{Sgs}-8$.

Construction of plasmids containing the cis-acting sequences and reporter genes. Plasmids of the pGGL series were constructed by cloning sequences from the intergenic region between $S g s-7$ and $S g s-8$ into the $S a l I$ site of pGLO-1 after they had been flanked with synthetic SalI linkers. pGLO-1 is pUC18 that contains the $S g s-3-l a c Z$ fusion gene (57) with 130 bp of $S g s-3$ upstream sequence. The $S a l$ site is at -130 . The number in the plasmid name is the amount of cis-acting sequence present (in base pairs); the number in parentheses gives the orientation of the sequence. For example, (7) means that the reporter gene is in the same orientation as the endogenous $\mathrm{Sgs}-7$. Plasmid pCSA-400(8) was constructed by cloning the 194-bp cis-acting sequences from $\mathrm{Sgs}-7 / \mathrm{Sgs}-8$ via SalI linkers into the $S a l$ site of pSA-400. pSA-400 is pUC18 containing an $S g s-4-A d h$ transcriptional fusion gene and 400 bp of 5'-flanking $S g s-4$ sequences. Plasmids of the pCA series were constructed by ligating sequences from the intergenic region (in one case together with stuffer sequences from pUC18) with attached SalI linkers to a synthetic SalI site 56 bp upstream from the D. melanogaster Adh gene in plasmid pNS1186 (obtained from N. Shen, Rutgers University). Germ line transformation vectors of the pCAX series were obtained by cutting out the relevant sequences of the pCA plasmid, filling in the ends with the Klenow fragment of $E$. coli DNA polymerase I, and ligating the blunt ends to the unique $\mathrm{HpaI}$ site in the polylinker of Carnegie 20. Plasmids of the pGGAX series were obtained by attaching SalI linkers to the 194-bp cis-acting region and cloning it into the SalI site of pGOAX.130, which contains an $S g s-3-A d h$ fusion gene with $130 \mathrm{bp}$ of $S g s-3$ upstream sequence present (46). Three different plasmids were obtained: pGGAX-7, in which the fusion gene is in the same orientation relative to the cisacting sequence as is $S g s-7$ in the endogenous situation; 
pGGAX-8, in which the fusion gene is in the same orientation as is $S g s-8$; and pGGAX-88, which contains a tandem duplication of the cis-acting sequences in the $S g s-8$ orientation.

Drosophila strains and transformations. The host strain for injections was $A d h^{f n 6} c n ; r y^{502}$ (obtained from J. W. Posakony, University of California, San Diego). Germ line transformation was done by the standard method $(47,54)$, as the transposase-producing helper plasmid phs $\pi$ (54a) or p $\pi 25.7 \mathrm{wc}$ (22) was used. Transformants were identified in the $\mathrm{G} 1$ progeny of injected animals as $r y^{+}$flies. Standard genetic techniques using the balancer strain $T(2 ; 3) A t a / C y O$; TM3, Sb $r y^{R K} \operatorname{Ser}$ (constructed by M. A. Crosby) were applied to map, balance, and make homozygous the autosomal insertion events. Markers and balancers are described by Lindsley and Grell (28) and by Lindsley and Zimm (29-31). DNA gel blotting (53) was used to determine that each transformed line analyzed contained only one insertion. In some cases, in situ hybridization to polytene chromosomes was performed by using a slight modification of the method described by Pardue and Gall (44). Transformant lines were named according to the following convention: $\mathrm{Tf}$, to indicate a transformant line; the numeral in parentheses indicates the chromosomal location of the insertion event; followed by the name of the plasmid construct transposed into the chromosome. The transient expression assay was originally developed by Martin et al. (35). The procedure as used in this work followed the technique described by Roark et al. (46). A positive result in this assay was histochemical staining only in the salivary gland cells of third-instar larvae.

Expression assays. For analysis of transient expression, histochemical staining assays on glands or dissected larvae were used. $\beta$-Galactosidase staining was performed as described by Vijay Raghavan et al. (57). Staining of glands and tissues for ADH activity was performed as described by Roark et al. (46). The quantitative spectrophotometric assay of ADH enzyme activity was done by the method of Sofer and Ursprung (52) with modifications and details described by Roark et al. (46). The quantitative measurement of $\beta$-galactosidase enzyme activity was performed as following. One animal equivalent of the same extract as prepared for the ADH assay was added to $2 \mathrm{ml}$ of $\mathrm{Z}$ buffer (42) adjusted to $\mathrm{pH} 7.5$ and supplemented with $0.5 \mathrm{mM} 4$-methylumbelliferyl$\beta$-D-galactopyranoside, which was used as the fluorogenic substrate (21). The reaction mixtures were incubated at $37^{\circ} \mathrm{C}$ while fluorescence of the reaction product $4 \mathrm{MU}$ (4-methylumbelliferone) was read every $30 \mathrm{~s}$ for $10 \mathrm{~min}$. The fluorescence was detected by using a Kontron SFM 25 spectrofluorimeter; excitation wavelength was $365 \mathrm{~nm}$, and emission wavelength was $455 \mathrm{~nm}$. A standard curve was constructed by using the above solutions containing four known concentrations of $4 \mathrm{MU}$ and was used to convert the raw fluorescence value into picomoles of $4 \mathrm{MU}$. A laboratory software program (17a) running on a Commodore PC20 was used for data evaluation and to calculate linear regression lines from the data. Total protein in each extract was measured by the method of Lowry et al. (33). We define $1 \mathrm{U}$ of hybrid Sgs-8- $\beta$-galactosidase enzyme as the amount of enzyme that releases $1 \mathrm{pmol}$ of $4 \mathrm{MU}$ per min. Final data were compiled and averaged, and standard deviations were calculated by using Microsoft's Excel program.

\section{RESULTS}

Activities of $\mathbf{S g s - 7 - A d h}$ and $S g s-8-l a c Z$ fusion genes in germ line transformants. As enzyme activity produced by fusion
TABLE 1. Activity of $S g s-7-A d h$ and $S g s-8-l a c Z$ fusion genes in germ line transformants

\begin{tabular}{|c|c|c|}
\hline \multirow{2}{*}{ Transformant } & \multicolumn{2}{|c|}{ 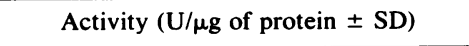 } \\
\hline & ADH & $\beta$-Galactosidase \\
\hline $\operatorname{Tf}(2) G L A X 1.0-2$ & $25.3 \pm 3.0$ & $3.09 \pm 0.82$ \\
\hline Tf(3)GLAX 1.0-3 & $30.2 \pm 5.6$ & $4.07 \pm 0.95$ \\
\hline Tf(3)GLAX 1.0-7 & $34.7 \pm 6.2$ & $4.62 \pm 1.23$ \\
\hline Avg & 30.1 & 3.93 \\
\hline
\end{tabular}

genes cannot be quantitatively compared with protein amounts of endogenous glue proteins, we have determined the activities of $S g s-7-A d h$ and $S g s-8-l a c Z$ fusion genes with their intact intergenic regions in germ line transformants, and we refer to these values as $100 \%$ expression of the genes. Three independent homozygous lines carrying the pGLAX1.0 construct (Fig. 1) were analyzed for ADH and $\beta$-galactosidase activity in salivary glands of third-instar larvae. For each line, three separate measurements were made, and the data were averaged and expressed as units of enzyme activity per microgram of total salivary gland protein (Table 1). The average of $\mathrm{ADH}$ activity for all three lines is $30.0 \mathrm{U} / \mu \mathrm{g}$. We take this to represent $100 \%$ expression of the $S g s-7-A d h$ gene. The average of $\beta$-galactosidase activity in the three lines is $3.9 \mathrm{U} / \mu \mathrm{g}$. We take this to represent $100 \%$ expression of the $S g s-8-l a c Z$ fusion gene.

The time of fusion gene expression in Tf()GLAX1.0 salivary glands was analyzed by histochemical staining of glands from feeding early- to mid-third-instar larvae, wandering third-instar larvae, and very late third-instar larvae with bloated salivary glands. ADH and $\beta$-galactosidase activities of early- to mid-third-instar salivary glands were variable, ranging from uniform and dark staining to nonuniform and light staining. Staining of glands taken from larvae in the second time period was uniformly distributed in the secretory cells of the glands. In the third group, $\beta$-galactosidase activity was found inside the lumens and ducts of the salivary glands but not within the cells of the glands themselves. In contrast, the cells of very late salivary glands continue to stain for ADH but at apparently reduced levels. This observation is consistent with the kinds of fusion genes used. $S g s-7-A d h$ is a transcriptional fusion in the 5 ' untranslated leader of both genes and therefore produces native ADH protein that lacks a signal peptide for transport into the lumen (9). In contrast, $S g s-8-l a c Z$ is a translational fusion with the coding sequences for 51 amino acids from $S g s-8$ present and contains the signal peptide necessary for secretion into the lumen of the gland. When third-instar larval carcasses or adults of the transformant strains were assayed for ADH activity, no enzyme activity could be detected in any other tissue. Histochemical staining for $\beta$-galactosidase of visceral tissue from transformants showed activity only in the midgut, which is found also in wild-type Drosophila strains and could be attributed to the Drosophila-encoded enzyme or to the enzyme from symbiotic bacteria in the gut.

The interaction of the fusion genes with the trans-regulating mutation l(l)npr-l was also analyzed. This mutation is a late larval lethal mutation that fails to pupariate $(23,24)$. In salivary glands of hemizygous mutant third-instar larvae, several phenotypes are noted: failure of intermolt puff regression and of ecdysterone-inducible puff formation (2); absence of accumulation of $68 \mathrm{C}$ glue protein gene transcripts; and absence of $68 \mathrm{C}$ glue gene RNA synthesis (10). Virgin females of the genotype y l(1)npr $w$ mal/Binsn were 


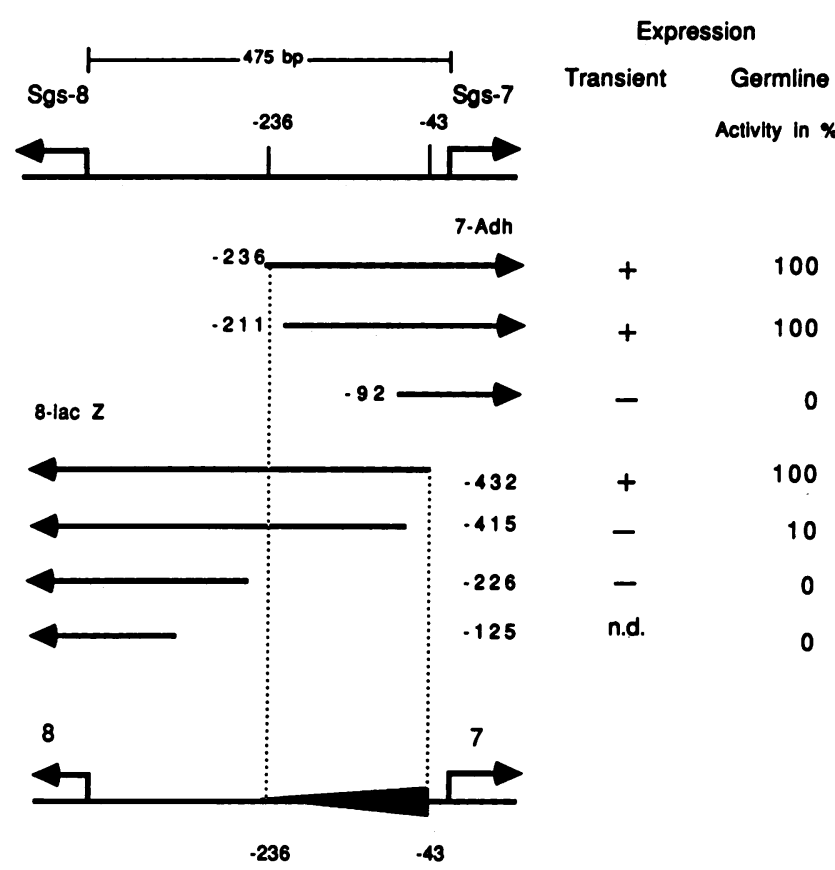

FIG. 2. Constructs containing the Sgs-7-Adh fusion gene or the Sgs-8-lacZ fusion gene with different amounts of 5'-flanking DNA. Expression of the fusion genes obtained in the transient assay or in germ line transformants is shown as either + or - or as percentage of enzyme activity compared with the expression of pGLAX1.0 transformants. Arrows show direction of transcription; numbers at the $5^{\prime}$ sites of fusion genes are upstream base pairs present. The wedge shows the orientation of cis-acting sequences at the endogenous locus. The thick end is near Sgs-7 in wild-type flies, and the thin end is near $S g s-8$. The boundaries at -236 and -43 show the fragment used for further tests.

mated to males of the three autosomal Tf()GLAX1.0 lines and to $A d h^{f 6} \mathrm{cn} ; \mathrm{ry}^{502}$ control males. Progeny male larvae hemizygous for the $y$ l(l)npr-l w mal chromosome were recognized by their white Malpighian tubules and yellow mouth hooks. Glands from these larvae and from their Binsn siblings were dissected and stained. No histochemical staining for either $\beta$-galactosidase or $\mathrm{ADH}$ could be detected in salivary glands of hemizygous $l(1) n p r-1$ males, while glands from control males that were hemizygous $l(l) n p r-l^{+}$and heterozygous for the pGLAX1.0 insertion stained for both enzymes.

Analysis of expression of Sgs-7-Adh and Sgs-8-lacZ fusion genes with various amounts of upstream sequences present. To test for the minimal amount of upstream sequence that has to be present to give full expression of the $\mathrm{Sgs-7}$ gene, the pGAX plasmid series was constructed (Materials and Methods; Fig. 1 and 2), and constructs with 236, 211, and 92 bp of upstream sequences present were tested in the transient assay, in a somatic transformation assay (described in Materials and Methods), and following germ line transformation. The transient assay was positive for the first two constructs and negative when only $92 \mathrm{bp}$ were present. For each construct, three independent autosomal transformant lines were quantitatively analyzed by determining the ADH enzyme activity in salivary gland extracts (Table 2). When either 236 or $211 \mathrm{bp}$ of $5^{\prime}$-flanking sequence are present, expression of the $S g s-7-A d h$ fusion gene is essentially $100 \%$; when only $92 \mathrm{bp}$ of upstream sequence are present, no
TABLE 2. Expression of $S g s-7-A d h$ with various amounts of upstream sequences

\begin{tabular}{|c|c|c|c|}
\hline $\begin{array}{c}\text { Upstream } \\
\text { bp } \\
\text { present }\end{array}$ & $\begin{array}{l}\text { Staining in } \\
\text { transient } \\
\text { assay }\end{array}$ & $\begin{array}{l}\text { Germ line } \\
\text { transformant }\end{array}$ & $\begin{array}{c}\text { ADH activity } \\
(\mathrm{U} / \mu \mathrm{g} \text { of } \\
\text { protein } \pm \mathrm{SD})\end{array}$ \\
\hline \multirow[t]{4}{*}{236} & \multirow[t]{4}{*}{+} & $\mathrm{Tf}(2) \mathrm{GAX} 0.26-1$ & $16.8 \pm 3.2$ \\
\hline & & $\mathrm{Tf}(3) \mathrm{GAX} 0.26-2$ & $20.1 \pm 3.3$ \\
\hline & & $\mathrm{Tf}(3) \mathrm{GAX} 0.26-3$ & $22.8 \pm 4.1$ \\
\hline & & Avg & 19.9 \\
\hline \multirow[t]{4}{*}{211} & \multirow[t]{4}{*}{+} & $\mathrm{Tf}(2) \mathrm{GAX} 0.24-1$ & $20.3 \pm 8.0$ \\
\hline & & $\mathrm{Tf}(3) \mathrm{GAX} 0.24-2$ & $36.4 \pm 4.4$ \\
\hline & & $\mathrm{Tf}(3) \mathrm{GAX} 0.24-4$ & $33.3 \pm 3.2$ \\
\hline & & Avg & 30.0 \\
\hline \multirow[t]{4}{*}{92} & \multirow[t]{4}{*}{-} & $\mathrm{Tf}(2) \mathrm{GAX} 0.12-1$ & $0.02 \pm 0.005$ \\
\hline & & $\mathrm{Tf}(2) \mathrm{GAX} 0.12-2$ & $0.06 \pm 0.02$ \\
\hline & & Tf(2)GAX0.12-3 & $0.01 \pm 0.02$ \\
\hline & & Avg & 0.03 \\
\hline
\end{tabular}

enzyme activity is detected. In RNA gel blot experiments, the transformants carrying the 92-bp construct lacked salivary gland ADH RNA (data not shown; 15). Histochemical staining of visceral tissues of larval carcasses and of adult flies showed no enzyme activity in all transformant lines. The results of these experiments show that the sequences required in cis for correct tissue and quantity of expression in the Sgs-7-Adh fusion gene test system include those between -211 and -92 bp relative to the start of $\mathrm{Sgs}^{-7}$ gene transcription.

The test for the minimal amount of upstream sequence required for correct functioning of $S g s-8$ was performed with the pGLX plasmid series (Fig. 1 and 2). Constructs with 432, $415,298,226$, and 125 bp of upstream sequence present were analyzed in the transient assay, via P-element transformation, or both (Table 3 ). As assayed by the transient procedure, the plasmid containing the 432-bp 5'-flanking DNA

TABLE 3. Expression of $S g s-8-l a c Z$ with various amounts of upstream sequences

\begin{tabular}{|c|c|c|c|}
\hline $\begin{array}{c}\text { Upstream } \\
\text { bp } \\
\text { present }\end{array}$ & $\begin{array}{l}\text { Staining in } \\
\text { transient } \\
\text { assay }\end{array}$ & $\begin{array}{l}\text { Germ line } \\
\text { transformants }\end{array}$ & 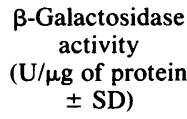 \\
\hline \multirow[t]{6}{*}{432} & + & $\operatorname{Tf}(3) G L X 0.68-1$ & $2.59 \pm 0.71$ \\
\hline & & $\operatorname{Tf}(2) G L X 0.68-2$ & $2.37 \pm 0.85$ \\
\hline & & $\operatorname{Tf}(2)$ GLX X0.68-3 & $3.48 \pm 0.51$ \\
\hline & & Tf(2)GLX0.68-4 & $4.11 \pm 1.02$ \\
\hline & & $\operatorname{Tf}(3) \mathrm{GLX} 0.68-5$ & $4.89 \pm 0.93$ \\
\hline & & Avg & 3.49 \\
\hline \multirow[t]{6}{*}{415} & - & $\operatorname{Tf}(3) \mathrm{GLX} 0.66-1$ & $0.18 \pm 0.04$ \\
\hline & & Tf(2)GLX0.66-2 & $0.31 \pm 0.12$ \\
\hline & & Tf(2)GLX0.66-3 & $0.21 \pm 0.06$ \\
\hline & & Tf(3)GLX0.66-4 & $0.13 \pm 0.03$ \\
\hline & & Tf(3)GLX0.66-5 & $0.43 \pm 0.08$ \\
\hline & & Avg & 0.25 \\
\hline 298 & - & $\mathrm{ND}^{a}$ & \\
\hline \multirow[t]{4}{*}{226} & - & Tf(3)GLX226-1 & $0.01 \pm 0.002$ \\
\hline & & Tf(3)GLX226-2 & $0.03 \pm 0.015$ \\
\hline & & Tf(3)GLX226-3 & $0.016 \pm 0.010$ \\
\hline & & Avg & 0.019 \\
\hline \multirow[t]{5}{*}{125} & ND & Tf(3)GLX125-1 & $0.08 \pm 0.05$ \\
\hline & & Tf(3)GLX125-2 & $0.018 \pm 0.07$ \\
\hline & & Tf(2)GLX125-3 & $0.035 \pm 0.06$ \\
\hline & & Tf(1)GLX125-4 & $0.05 \pm 0.02$ \\
\hline & & Avg & 0.046 \\
\hline
\end{tabular}

${ }^{a}$ ND, Not determined. 
sequence gave staining; none of the other constructs exhibited any $\beta$-galactosidase activity. Germ line transformants carrying four of the five deletion constructs were established, and $\beta$-galactosidase activities were measured in salivary gland extracts of climbing third-instar larvae from transformation-homozygous lines. When 432 bp of upstream sequences are present, the expression of $S g s-8-l a c Z$ is $100 \%$, but expression drops to $10 \%$ when 415 bp are present. In transformants carrying Sgs-8-lacZ fusion genes with 226 or 125 bp of 5 -flanking sequences, no enzyme activity could be detected. Again, staining of other tissues and stages showed no enzyme activity for all the transformed lines except for the background staining in the midgut. These results show that sequences located asymmetrically in the intergenic region are required for correct expression of both $S g s-7$ and $S g s-8$, as outlined in Fig. 2. The overlapping sequences required for expression of both genes are referred to as the cis-acting element and were further tested for their regulatory capacity.

The cis-acting element can enhance the expression of other glue genes. The putative cis-acting region was tested for its capacity to restore normal quantity of expression of an $\mathrm{Sgs}-3$ or $S g s-4$ gene construction lacking its own quantitative control sequences. The 194-bp region between -236 and -43 relative to $S g s-7$ was linked to an $S g s-3-l a c Z$ fusion gene with $130 \mathrm{bp}$ of $5^{\prime}$-flanking sequence. This gene construction without the 194-bp region (pGLO-1) is negative in the transient assay but gives faint histochemical staining in salivary glands of germ line transformant third-instar larvae (57). In our experiments, none of 14 third-instar larvae showed staining in the salivary glands after injection of pGLO-1 into the embryos, whereas 6 of 9 larvae showed staining in the salivary glands after injection of pGGL194(7), in which the cis-acting element is placed in the Sgs-7 orientation in front of the -130-bp Sgs-3-lacZ gene. With plasmid pGGL-194(8), in which the element is placed in the $S g s-8$ orientation, we obtained 14 positives out of 36 larvae analyzed in the transient assay (Fig. 3). For testing the cis-acting element for an enhancing effect on expression of $S g s-4$, we linked the 194-bp region to a transcriptional $S g s-4-A d h$ fusion gene with 400 bp of $S g s-4$ upstream sequence present (pSA-400). An Sgs-4 gene with 398 bp of upstream sequence has been shown to be completely inactive in germ line transformants (38). In our transient assays with pSA-400, none of 18 larvae showed histochemical staining in their salivary glands, whereas with pCSA-400(8), in which the cis-acting element is located at $-400 \mathrm{bp}$ of the $S g s-4-A d h$ fusion gene in $S g s-8$ orientation, we obtained 3 out of 26 positively reacting larvae (Fig. 3). These results show that the cis-acting element can enhance expression of other glue genes and can replace the enhancers ordinarily found upstream of these genes.

It is not possible to draw conclusions about quantitative expression from the transient assays. The number of individual larvae or the number of cells in the salivary glands that stain positively depends apparently on the uptake of plasmid DNA by the salivary gland precursor cells in the injected embryo and not on any quality of the promoter tested. Therefore, germ line transformation via P-element vectors was used to quantify the enhancing effect of the cis-acting element. The 194-bp region was cloned into the SalI site at -130 of GOAX.130, a construct containing an Sgs-3-Adh fusion gene with 130 bp of 5'-flanking sequence (46). Three different derivatives were tested in the germ line: pGGAX-7, in which the reporter gene is in the same orientation relative to the cis-acting element as is $\mathrm{Sgs-7}$ in the
A

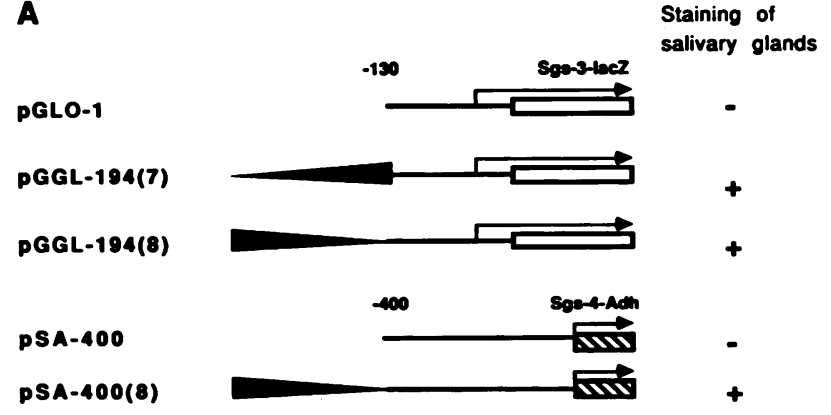

$\mathbf{B}$

Adh activity in salivary glands

GOAX.13

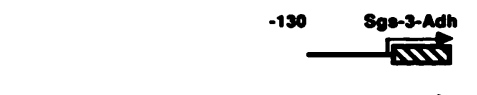

1

GGAX-7

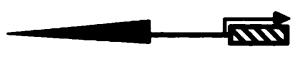

90

GGAX-8

GGAX-88

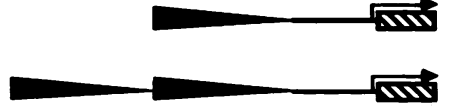

27

46

FIG. 3. Constructs used in the transient expression procedure (A) and in germ line transformation analysis (B). Open bars, $E$. coli lac $Z$ coding sequences; striped bars, D. melanogaster $A d h$ coding sequences; thin lines, upstream sequences of $\mathrm{Sgs}-3$ and $\mathrm{Sgs}-4$ (arrows indicate direction of transcription); wedge-shaped line, cis-acting sequences from -236 of $\mathrm{Sgs-7}$ (thin end) to -43 of $\mathrm{Sgs-7}$ (thick end). For nomenclature of plasmids, see Materials and Methods.

endogenous situation; pGGAX-8, in which the reporter gene is in the $S g s-8$ orientation; and pGGAX-88, a tandem insertion of the cis-acting element in $\mathrm{Sgs}-8$ orientation (Fig. 3). ADH enzyme activities in extracts from salivary glands of different transformant lines were determined (Table 4) and compared with the enzyme activity of the $S g s-3-A d h$ fusion

TABLE 4. ADH activity in GGAX germ line transformants

\begin{tabular}{|c|c|}
\hline Transformant line & $\begin{array}{l}\text { ADH activity } \\
(\mathrm{U} / \mu \mathrm{g} \text { of } \\
\text { protein } \pm \mathrm{SD})\end{array}$ \\
\hline \multirow{3}{*}{\multicolumn{2}{|c|}{ 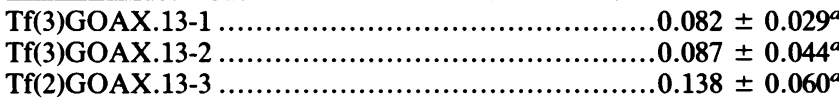 }} \\
\hline & \\
\hline & \\
\hline Avg ................ & .. 0.102 \\
\hline $\operatorname{Tf}(3) \mathrm{GGAX}-7-1$. & $6.45 \pm 0.98$ \\
\hline Tf(2)GGAX-7-2. & $. .13 .71 \pm 2.82$ \\
\hline Tf(3)GGAX-7-3... & .. $7.89 \pm 2.22$ \\
\hline \multicolumn{2}{|l|}{$\mathrm{Tf}(2) \mathrm{GGAX}-7-4}$. \\
\hline Avg ................... & 8.99 \\
\hline Tf(2)GGAX-8-1. & .. $3.77 \pm 1.23$ \\
\hline Tf(3)GGAX- 8 & $3.69 \pm 0.98$ \\
\hline Tf(2)GGAX-8-3... & $1.89 \pm 0.61$ \\
\hline \multirow{2}{*}{\multicolumn{2}{|c|}{$\begin{array}{r}\text { Tf(3)GGAX-8-4... } \\
\text { Avg } \ldots \ldots \ldots \ldots \ldots \ldots\end{array}$}} \\
\hline & 2.75 \\
\hline \multicolumn{2}{|l|}{ Tf(2)GGAX-88-1 . } \\
\hline \multicolumn{2}{|c|}{ 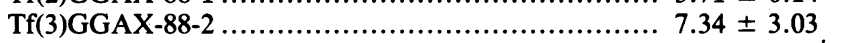 } \\
\hline \multicolumn{2}{|c|}{ Tf(3)GGAX-88-3 …........................................ $2.76 \pm 0.42^{b}$} \\
\hline Avg. & 4.61 \\
\hline
\end{tabular}

${ }^{a}$ Data from Roark et al. (46).

$b$ Values from homozygous lethals were doubled after it was ascertained that the lethal effect is before third larval instar ( 20 larvae from each line were tested for heterozygosity in polytene chromosome squashes). 


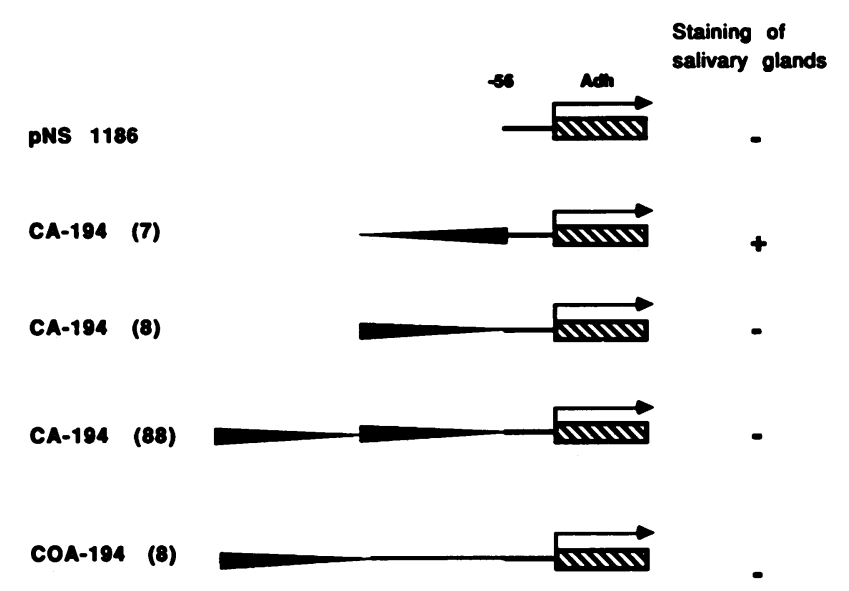

FIG. 4. Constructs tested in the transient assay. Striped bars, $D$. melanogaster Adh coding sequences; thick lines, Adh promoter sequences; thin line, $200 \mathrm{bp}$ from pUC18 (stuffer sequence); wedgeshaped line, cis-acting sequences from -236 of $\mathrm{Sgs}^{-7}$ (thin end) to -43 of $S g s-7$ (thick end). For nomenclature of plasmids, see Materials and Methods.

gene without the cis-acting element from $S g s-7 / S g s-8$ in the GOAX.130 lines (data reported by Roark et al. [46]). In larvae transformed with the GGAX-7 construct, salivary gland activity of $\mathrm{ADH}$ is increased 90 -fold relative to Tf()GOAX.130 lines. In the Tf()GGAX-8 larvae, carrying the cis-acting element in the opposite orientation, the enhancing effect is 27-fold. A tandem duplication of the cis-acting element in the $S g s-8$ orientation almost doubles the activity of the reporter gene relative to the similar construct with a single copy (Fig. 3).

The cis-acting element shows a directional tissue- and stagespecific regulatory function. To test the cis-acting element for its capacity to confer the Sgs gene expression pattern on a heterologous promoter, plasmids of the CA series were constructed. The 194-bp region was fused to an $A d h$ gene truncated at -56 bp upstream of the larval proximal promoter. Three different clones were obtained: pCA-194(7), with the Adh gene in the $S g s-7$ orientation; pCA-194(8), with the $A d h$ gene in the $S g s-8$ orientation; and pCA-194(88), with a tandem duplication of the cis-acting element in the $S g s-8$ orientation (Fig. 4). The three constructions were tested in the transient assay, and only pCA-194(7) gave a positive result, with 5 of 21 third-instar larvae showing $\mathrm{ADH}$ activity in the salivary glands. pCA-194(8) and pCA-194(88) were negative, with none of 27 and none of 17 larvae, respectively, showing staining. These results indicate that the 194-bp region contains the regulatory element for stage- and tissue-specific expression. One possible reason for the negative results obtained with plasmids of the $S g s-8$ orientation could be that the lower-level expression observed in the GGAX-8 lines is below the threshold at which expression can be monitored in the transient assay. To test this possibility, we transferred the pCA-194(8) construct into the P-element vector Carnegie 20 and performed germ line transformation. Salivary glands from larvae of the resulting Tf()CAX-194(8) lines were tested in the ADH histochemical assay, but no staining was observed. Another explanation for the nonexpression in the $S g s-8$ orientation could be that the element in this orientation can work only when it is placed at the same distance from the Adh gene as it is from $S g s-8$ in the endogenous situation. This possibility was tested in the transient assay by injecting plasmid pCOA194(8), which has a 190-bp stuffer sequence from pUC18, between the cis-acting element and the start of the Adh gene. None of 38 third-instar larvae tested in the transient assay showed staining in the salivary glands.

Further delimitation of the cis-acting element. The 194-bp region was split into two segments, a 86-bp segment from -48 to -133 (relative to $S g s-7$ ) and a 99-bp segment from -138 to -236 . Both fragments were fused to the $S g s-3-l a c Z$ gene with $130 \mathrm{bp}$ of $5^{\prime}$-flanking sequences in pGLO-1, and plasmids pGGL-86(8) and pGGL-99(7) were obtained. Only pGGL-86(8) gave a positive result when tested in the transient assay, with 9 of 37 larvae showing staining in their salivary glands. With pGGL-99(7), none of 35 larvae showed staining in their salivary glands. This result shows that at least the enhancing or amplifying effect on glue gene expression is mediated by the $86-\mathrm{bp}$ portion of the $S g s-7 / S g s-8$ cis-acting sequences located at -48 to -133 relative to Sgs-7.

Sequence homologies at $68 \mathrm{C}$. At least three regulatory elements required for full expression of $S g s-3$ have been identified: the proximal element (at -56 to -98 ), the distal element (at -491 to -629 ), and one or more remote upstream elements $(34,46)$. Roark et al. (46) have shown that the proximal and distal elements are functionally equivalent, each acting to direct correct tissue- and stage-specific expression at low levels. When combined, these elements act synergistically to give high levels of expression. We have shown in this work that the $S g s-7 / S g s-8$ cis-acting element is capable of functioning as an enhancer when present with the proximal element of $S g s-3$. This finding suggests that it is also functionally equivalent to this element and may have a similar regulatory sequence. Todo et al. (55) identified single-base-pair substitutions at several sites in the proximal element that inactivate it. Aligning the sequences of the proximal $S g s-3$ element, the distal $S g s-3$ element, and the Sgs-7/Sgs-8 cis-acting element reveals a similar sequence in each of the three elements. The relevant sequences are shown in Fig. 5, with the common sequences boxed. Note that within the $S g s-7 / S g s-8$ cis-acting element, there are two copies of the homology present, both facing $S g s-7$, and they are located in the 86-bp portion of the cis-acting element that was shown by our experiments to contain the regulatory sequences.

\section{DISCUSSION}

Regulatory sequences for $\mathbf{S g s - 7}$ and $\mathbf{S g s - 8}$ are not separate for each gene and are located closer to Sgs-7. The fact that there are homologous sequences present in the base pairs immediately upstream of both the $S g s-7$ and $S g s-8$ genes suggests that each of these sequences is solely required separately for correct expression of the gene close to it. This is the case for $S g s-7$ but not for $S g s-8$, as reported in this work. Sequences located closer to $\mathrm{Sgs}-7$ are required for correct functioning of both $\mathrm{Sgs-7}$ and $\mathrm{Sgs}-8$ genes.

The cis-acting element of the $S g s-7 / S g s-8$ intergenic region as defined in this work regulates the correct expression of Sgs-7. It is also necessary for full expression of $S g s-8$. We cannot exclude that sequences located between the transcription start site of $S g s-8$ and the cis-acting element are additionally necessary for correct functioning of $S g s-8$ for two reasons. First, the cis-acting element does not confer its enhancing capability equivalently in both orientations. In the Tf()GGAX transformant lines, the enhancing effect of the cis-acting element in the $S g s-8$ orientation is only one-third 


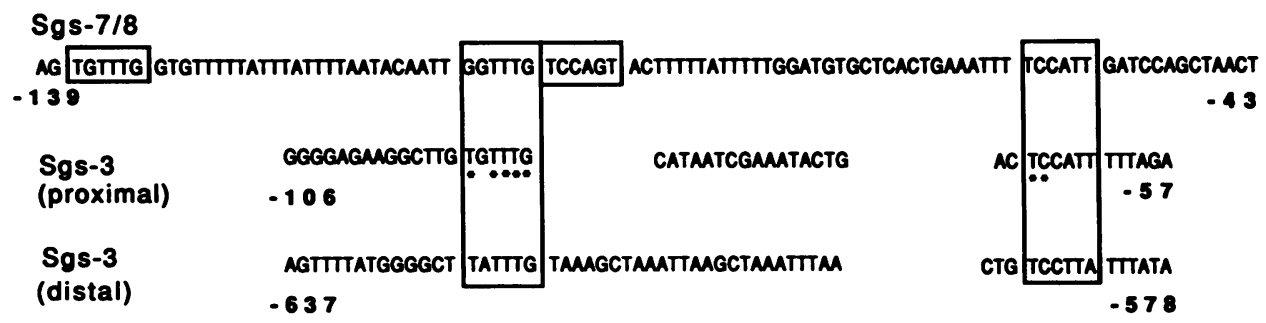

FIG. 5. Comparison of the sequence of the cis-acting elements of $S g s-7 / S g s-8$ with the sequences of the two functionally equivalent elements of $S g s$-3. The elements show boxes of homology with an AT-rich spacer region between them. Nucleotides indicated by asterisks have been shown to be important by single-base substitution mutation (55). Blank spaces between sequences were inserted where needed to allow alignment. Numbering is from the start site of $S g s-3$ or $S g s-7$.

of the effect found in the $S g s-7$ orientation. A similar quantitative difference due to orientation has been observed for the distal element of Sgs-3 (46). Second, the cis-acting element cannot confer stage- and tissue-specific expression on a heterologous promoter in the $S g s-8$ orientation, although it can in the $S g s-7$ orientation. We have excluded one possible explanation for this phenomenon, that the element in the $S g s-8$ orientation can work only when it is placed at a distance to a reporter gene that reflects the original distance from $S g s-8$. This possibility is ruled out by the negative results of the transient expression assay using a plasmid in which the cis-acting element was placed in about the same distance to the Adh gene as it is to $S g s-8$ in the endogenous situation. Another explanation is that the sequences necessary for enhancing the activity of a stage- and tissue-specific element are less than those necessary to actually be a stageand tissue-specific element. One conclusion that might be drawn from these experiments is that there may be sequences near $S g s-8$ (and also within the 400 bp 5' of $S g s-4$ ) that are not sufficient to confer any expression on the adjacent gene but in combination with a potent enhancer (and the proteins binding there) give rise to normal expression. In the case of $S g s-8$, a possible explanation could be that the sequences nearer to $S g s-8$ have changed to weaker binding sites for salivary gland-specific trans-acting factors. Expression of the $S g s-8$ gene continues because of the proximity of the $\mathrm{Sgs}-7$ regulating sequences, in combination with the weak sites. If $S g s-7$ regulatory sequences were not so close, then $S g s-8$ might be a transcriptionally quiescent pseudogene.

Interaction with the mutation $l(1)$ npr-1. The fact that the expression of the two glue protein fusion genes in the Tf()GLAX1.0 transformant lines is dependent on the l(l)npr- $1^{+}$gene product suggests three conclusions. First, the process that $l(1) n p r-1^{+}$regulates is promoter specific, since the $A d h$ gene, with its proximal promoter that functions in a variety of larval tissues, directs the accumulation of substantial amounts of ADH activity in the fat body, midgut, and gastric cecae of $l(1) n p r-l$-mutant larvae (15). Second, the process of $68 \mathrm{C}$ glue protein gene expression controlled (either directly or indirectly) by the $l(l) n p r-1^{+}$ gene product is most likely to be gene transcription. Expression of the $S g s-7-A d h$ fusion gene, which contains only 25 nucleotides of the glue protein gene transcription unit, requires the $l(l) n p r-1^{+}$gene product. Any model for $l(1) n p r-1^{+}$function that requires a sequence-specific interaction with the body of the $S g s-7$ glue protein gene or of its primary transcript (the intervening sequence, for example) is untenable. Third, at $68 \mathrm{C}$ there are a minimum of two sites of action for $l(1) n p r-l^{+}$, since the sequences required for cor- rect stage- and tissue-specific expression of $S g s-3$ also retain the requirement for this genetic function (57).

Divergently transcribed gene pairs and shared enhancers. At least three different kinds of divergently transcribed gene pairs are known. One is the type that has both genes expressed in a single tissue at a single time during development, like the $S g s-7 / S g s-8$ gene pair. These depend on a single relatively small region that functions bidirectionally to specify tissue and stage of gene expression. The second kind, for example, the yolk protein-1/yolk protein-2 (Yp-1) $Y p$-2) gene pair, has both genes expressed in two different tissues at a single time during development. The third kind, like the Sgs-4/Pig-1 gene pair, has both genes expressed within a single tissue but at different times during development $(8,20)$. The $Y p-1$ and $Y p-2$ genes are expressed in two tissues of the adult female: the ovarian follicle cells and the fat body (6). They are divergently transcribed, with a 1,225-bp intergenic spacer between the $5^{\prime}$ ends (13). The intergenic region contains an enhancer conferring fat-bodyspecific expression located adjacent to $Y p-l$ and two enhancers for expression in ovarian follicle cells located within and adjacent to $Y p-2$ (13). These DNA sequences function in a bidirectional manner when positioned near reporter genes $(14,32)$. One of the follicle cell specificity elements resides within the first exon of $Y p-2$ (32). The follicle cell specificity element, located 43 to 343 bp upstream of $Y p-2$, in the intergenic region, has multiple components. These include both positive-acting and negative-acting sequences that serve to regulate yolk protein gene transcription in spatially distinct subpopulations of follicle cells. The $\mathrm{Sgs}-7 / \mathrm{Sgs}-8$ gene pair is a simplified example of the regulatory sequence arrangement seen in the $Y p-1 / Y p-2$ gene pair: expression in one tissue and one bidirectional regulatory element.

Bidirectional control by a shared enhancer is reported for at least two additional gene pairs. Expression of chicken $\beta$ and $\varepsilon$-globin genes in erythroid cells is controlled by a shared enhancer which is located about $1.6 \mathrm{~kb} \mathrm{3}$ of the $\beta$ promoter and about $1.5 \mathrm{~kb} 5^{\prime}$ of the $\varepsilon$ promoter (43). The inverted orientation of this enhancer leads to 60 to $80 \%$ activity of an adjacent reporter gene. The murine $\alpha 1$ (IV) and $\alpha 2$ (IV) collagen chain genes are divergently transcribed and separated by $130 \mathrm{bp}$. Transcription of these genes is regulated by a bidirectional promoter located in the intergenic region and a shared enhancer located in the first intron of the $\alpha 1$ (IV) chain gene (7). These genes are coordinately regulated by their enhancers.

Comparison with other glue genes. The upstream sequences of $S g s-3, S g s-4, S g s-5, S g s-7$, and $S g s-8$ have been determined $(15,20,50)$. A search for common sequence motifs within the regulatory regions of these genes revealed 
regions of homology between -90 and -76 bp of $S g s-3$, between -137 and -123 bp of $S g s-4$, between -116 and -105 bp of $S g s-5$, and in the conserved regions upstream of $S g s-7(-93$ to -48$)$ and $S g s-8(-91$ to -44$)$. In all cases, the region of homology is located within sequences required for correct expression of the adjacent glue gene $(19,51,57$; this work). The most striking evidence for a regulatory function of conserved sequences in the upstream region of glue genes comes from the work of Todo et al. (55); they first show which single base pairs in the proximal element of $\mathrm{Sgs}-3$ are absolutely required for correct function and then find these base pairs to be located among conserved sequence regions $5^{\prime}$ of all sequenced glue genes. Todo et al. (55) found that a functional $S g s-3$ regulatory element contains a TNTTTG homology and a TCCAT(T/A) homology separated by a certain number of nucleotides. There are two of these elements located in the 86-bp cis-acting region of $S g s-7 / S g s-8$ described in this work in an interspersed array facing Sgs-7. We know that at least the one closer to $S g s-7$ is necessary for correct expression of $\mathrm{Sgs}-8$, because the deletion of $17 \mathrm{bp}$ that reduces $S g s-8$ expression 10 -fold removes the TCCATT box of this element.

\section{ACKNOWLEDGMENTS}

We thank C. A. Mayeda for assisting with the microinjection of Drosophila embryos and M. F. Yanofsky for the sequence determination of BAL 31 deletion endpoints. We also thank B. A. Hamilton and P. H. Mathers for critical reading of different drafts of the manuscript. We are grateful also to the following individuals: J. J. Bonner, V. Pirrotta, and G. M. Rubin for providing cloned DNA molecules, in some cases prior to publication; and J. W. Posakony and B. T. Wakimoto for fly strains.

A.H. was supported by grant Ho1047/2-1 from the Deutsche Forschungsgemeinschaft. At the outset of this work, M.D.G. was supported by National Research Award 5 T32 GM07616, awarded by the National Institute of General Medical Sciences of the National Institutes of Health to the California Institute of Technology. The work was funded by grant GM28075 (to E.M.M.) from the National Institute of General Medical Sciences of the National Institutes of Health.

\section{REFERENCES}

1. Beckendorf, S. K., and F. C. Kafatos. 1976. Differentiation in the salivary glands of Drosophila melanogaster: characterization of the glue proteins and their developmental appearance. Cell 9:365-373.

2. Belyaeva, E. S., I. E. Vlassova, Z. M. Biyasheva, V. T. Kakpakov, G. Richards, and I. F. Zhimulev. 1981. Cytogenetic analysis of the 2B3-4-2B11 region of the X-chromosome of Drosophila melanogaster. II. Changes in $20-\mathrm{OH}$ ecdysone puffing caused by genetic effects. Chromosoma 84:207-219.

3. Benyajati, C., N. Spoerel, H. Haymerle, and M. Ashburner. 1983. The messenger RNA for alcohol dehydrogenase in Drosophila melanogaster differs in its $5^{\prime}$ end in different developmental stages. Cell 33:125-133.

4. Bonner, J. J., C. Parks, J. Parker-Thornburg, M. A. Mortin, and H. R. B. Pelham. 1984. The use of promoter fusions in Drosophila genetics: isolation of mutations affecting the heat shock response. Cell 37:979-991.

5. Bourouis, M., and G. Richards. 1985 . Remote regulatory sequences of the Drosophila glue gene $S g s-3$ as revealed by P-element transformation. Cell 40:349-357.

6. Brennan, M. D., A. J. Wiener, T. J. Goralski, and A. P. Mahowald. 1982. The follicle cells are a major site of vitellogenin synthesis in Drosophila melanogaster. Dev. Biol. 89:225236.

7. Burbelo, P. D., G. R. Martin, and Y. Yamada. 1988. $\alpha 1($ IV) and $\alpha 2$ (IV) collagen genes are regulated by a bidirectional promoter and a shared enhancer. Proc. Natl. Acad. Sci. USA 85:96799682.

8. Chen, C.-N., T. Malone, S. K. Beckendorf, and R. L. Davis. 1987. At least two genes reside within a large intron of the dunce gene of Drosophila. Nature (London) 329:721-724.

9. Crowley, T. E., M. W. Bond, and E. M. Meyerowitz. 1983. The structural genes for three Drosophila glue proteins reside at a single polytene chromosome puff locus. Mol. Cell. Biol. 3:623634.

10. Crowley, T. E., P. H. Mathers, and E. M. Meyerowitz. 1984. A trans-acting regulatory product necessary for expression of the Drosophila melanogaster 68C glue gene cluster. Cell 39:149156.

11. Crowley, T. E., and E. M. Meyerowitz. 1984. Steroid regulation of RNAs transcribed from the Drosophila $68 \mathrm{C}$ polytene chromosome puff. Dev. Biol. 102:110-121.

12. Fraenkel, G., and V. J. Brookes. 1953. The process by which the puparia of many species of flies become fixed to the substrate. Biol. Bull. 105:442-449.

13. Garabedian, M. J., M.-C. Hung, and P. C. Wensink. 1985. Independent control elements that determine yolk protein gene expression in alternative Drosophila tissues. Proc. Natl. Acad. Sci. USA 82:1396-1400.

14. Garabedian, M. J., B. M. Shepard, and P. C. Wensink. 1986. A tissue-specific transcription enhancer from the Drosophila yolk protein 1 gene. Cell 45:859-867.

15. Garfinkel, M. D. 1988. Structural and functional studies of the 68C glue protein gene cluster in Drosophila melanogaster. Ph.D. dissertation, California Institute of Technology, Pasadena.

16. Garfinkel, M. D., R. E. Pruitt, and E. M. Meyerowitz. 1983. DNA sequences, gene regulation and modular protein evolution in the Drosophila 68C glue gene cluster. J. Mol. Biol. 168:765789.

17. Giangrande, A., C. Mettling, and G. Richards. 1987. Sgs-3 transcript levels are determined by multiple remote elements. EMBO J. 6:3079-3084.

17a.Grams, R. Unpublished data.

18. Hansson, L., and A. Lambertsson. 1983. The role of $s u(f)$ gene function and ecdysterone in transcription of glue polypeptide mRNAs in Drosophila melanogaster. Mol. Gen. Genet. 192: 395-401.

19. Hofmann, A., A. Keinhorst, A. Krumm, and G. Korge. 1987. Regulatory sequences of the Sgs-4 gene of Drosophila melanogaster analysed by P-element mediated transformation. Chromosoma 96:8-17.

20. Hofmann, A., and G. Korge. 1987. Upstream sequences of dosage-compensated and non-compensated alleles of the larval secretion protein gene $\mathrm{Sgs}_{-4}$ in Drosophila. Chromosoma 96: $1-7$.

21. Jefferson, R. A., T. A. Kavanagh, and M. W. Bevan. 1987. GUS fusions: $\beta$-glucuronidase as a sensitive and versatile gene fusion marker in higher plants. EMBO J. 6:3901-3907.

22. Karess, R. E., and G. M. Rubin. 1984. Analysis of P transposable element functions in Drosophila. Cell 38:135-146.

23. Kiss, I., G. Bencze, E. Fekete, A. Fodor, J. Gausz, P. Maroy, J. Szabad, and J. Szidonya. 1976. Isolation and characterization of $X$-linked lethal mutants affecting differentiation of the imaginal discs in Drosophila melanogaster. Theor. Appl. Genet. 48:217226.

24. Kiss, I., J. Szabad, and J. Major. 1978. Genetic and developmental analysis of puparium formation in Drosophila. Mol. Gen. Genet. 164:77-83.

25. Korge, G. 1975. Chromosome puff activity and protein synthesis in larval salivary glands of Drosophila melanogaster. Proc. Natl. Acad. Sci. USA 72:4550-4554.

26. Korge, G. 1977. Larval saliva in Drosophila melanogaster: production, composition and relationship to chromosome puffs. Dev. Biol. 58:339-355.

27. Lane, N. J., Y. R. Carter, and M. Ashburner. 1972. Puffs and salivary gland function: the fine structure of the larval and prepupal salivary glands of Drosophila melanogaster. Wilhelm 
Roux' Archiv. Entwicklungsmech. Org. 169:216-238.

28. Lindsley, D. L., and E. H. Grell. 1968. The genetic variations of Drosophila melanogaster. Publication 627. Carnegie Institution of Washington, Baltimore.

29. Lindsley, D., and G. Zimm. 1985. In Drosophila Information Service 62.

30. Lindsley, D., and G. Zimm. 1985. In Drosophila Information Service 64.

31. Lindsley, D., and G. Zimm. 1985. In Drosophila Information Service 65.

32. Logan, S. K., and P. C. Wensink. 1990. Ovarian follicle cell enhancers from the Drosophila yolk protein genes: different segments of one enhancer have different cell-type specificities that interact to give normal expression. Genes Dev. 4:613-623.

33. Lowry, O. H., N. J. Rosebrough, A. L. Farr, and R. J. Randall. 1951. Protein measurement with the Folin phenol reagent. J. Biol. Chem. 193:265-275.

34. Martin, M., A. Giangrande, C. Ruiz, and G. Richards. 1989. Induction and repression of the Drosophila Sgs-3 glue gene are mediated by distinct sequences in the proximal promoter. EMBO J. 8:561-568.

35. Martin, P., A. Martin, A. Osmani, and W. Sofer. 1986. A transient expression assay for tissue-specific gene expression of alcohol dehydrogenase in Drosophila. Dev. Biol. 117:574-580.

36. Maxam, A. M., and W. Gilbert. 1977. A new method for sequencing DNA. Proc. Natl. Acad. Sci. USA 74:560-564.

37. Maxam, A. M., and W. Gilbert. 1980. Sequencing end-labeled DNA with base-specific chemical cleavages. Methods Enzymol. 65:499-560.

38. McNabb, S. L., and S. K. Beckendorf. 1986. Cis-acting sequences which regulate expression of the $\mathrm{Sgs}-4$ glue protein gene of Drosophila. EMBO J. 5:2331-2340.

39. Mestril, R., P. Schiller, J. Amin, H. Klapper, J. Ananthan, and R. Voellmy. 1986. Heat shock and ecdysterone activation of the Drosophila melanogaster hsp23 gene: a sequence element implied in developmental regulation. EMBO J. 5:1667-1673.

39a.Meyerowitz, E. M. Unpublished data.

40. Meyerowitz, E. M., and D. S. Hogness. 1982. Molecular organization of a Drosophila puff site that responds to ecdysone. Cell 28:165-176.

41. Meyerowitz, E. M., K. Vijay Raghavan, P. H. Mathers, and M. Roark. 1987. How Drosophila larvae make glue: control of Sgs-3 gene expression. Trends Genet. 3:288-293.

42. Miller, J. H. 1972. Experiments in molecular genetics. Cold Spring Harbor Laboratory, Cold Spring Harbor, N.Y.

43. Nickol, J. M., and G. Felsenfeld. 1988. Bidirectional control of the chicken $\beta$ - and $\varepsilon$-globin genes by a shared enhancer. Proc. Natl. Acad. Sci. USA 85:2548-2552.

44. Pardue, M. L., and J. G. Gall. 1975. Nucleic acid hybridization to the DNA of cytological preparations. Methods Cell Biol. 10:1-16.

45. Ramain, P., A. Giangrande, G. Richards, and M. Bellard. 1988. Analysis of a DNaseI-hypersensitive site in transgenic Drosophila reveals a key regulatory element of Sgs-3. Proc. Natl. Acad. Sci. USA 85:2718-2722.

46. Roark, M., K. Vijay Raghavan, T. Todo, C. A. Mayeda, and E. M. Meyerowitz. 1990. Cooperative enhancement at the Drosophila Sgs-3 locus. Dev. Biol. 139:121-133.

47. Rubin, G. M., and A. C. Spradling. 1982. Genetic transformation of Drosophila with transposable element vectors. Science 218:348-353.

48. Rubin, G. M., and A. C. Spradling. 1983. Vectors for P element mediated gene transfer in Drosophila. Nucleic Acids Res. 11:6341-6351.

49. Sanger, F., S. Nicklen, and A. R. Coulson. 1977. DNA sequencing with chain-terminating inhibitors. Proc. Natl. Acad. Sci. USA 74:5463-5467.

50. Shore, E. M., and G. M. Guild. 1986. Larval salivary gland secretion proteins in Drosophila. Structural analysis of the Sgs-5 gene. J. Mol. Biol. 190:149-158.

51. Shore, E. M., and G. M. Guild. 1987. Closely linked DNA elements control the expression of the $S g s-5$ glue protein gene in Drosophila. Genes Dev. 1:829-839.

52. Sofer, W., and H. Ursprung. 1968. Drosophila alcohol dehydrogenase: purification and partial characterization. J. Biol. Chem. 243:3110-3115.

53. Southern, E. M. 1975. Detection of specific sequences among DNA fragments separated by gel electrophoresis. J. Mol. Biol. 98:503-517.

54. Spradling, A. C., and G. M. Rubin. 1982. Transposition of cloned P elements into Drosophila germline chromosomes. Science 218:341-347.

54a.Steller, H., and V. Pirrotta. Unpublished data

55. Todo, T., M. Roark, K. Vijay Raghavan, C. A. Mayeda, and E. M. Meyerowitz. 1990. Fine structure mutational analysis of a stage- and tissue-specific promoter element of the Drosophila glue gene Sgs-3. Mol. Cell. Biol. 10:5991-6002.

56. Vieira, J., and J. Messing. 1987. Production of single-stranded plasmid DNA. Methods Enzymol. 153:3-11.

57. Vijay Raghavan, K., M. A. Crosby, P. H. Mathers, and E. M. Meyerowitz. 1986. Sequences sufficient for correct regulation of Sgs-3 lie close to or within the gene. EMBO J. 5:3321-3326. 